\title{
Influência de tratamentos para enxaqueca na qualidade de vida: revisão integrativa de literatura
}

\author{
Influence of treatments for migraine on quality of life: literature integrative review
}

Influencia de los tratamientos para la migraña en la calidad de vida: revisión integradora de la literatura

\author{
Thais Stefane', Anamaria Alves Napoleão", Fátima Aparecida Emm Faleiros Sousa"', Priscilla Hortense" \\ ' Universidade Federal de São Carlos, Departamento de Enfermagem, \\ Curso de Enfermagem. (Graduanda) São Carlos-SP, Brasil. \\ "Universidade Federal de São Carlos, Departamento de Enfermagem, Curso de Enfermagem. São Carlos-SP, Brasil. \\ "I' Universidade de São Paulo, Escola de Enfermagem, \\ Departamento de Enfermagem Geral e Especializada. Ribeirão Preto-SP, Brasil.
}

\author{
Submissão: 03-02-2011 Aprovação: 24-01-2012
}

\section{RESUMO}

Este estudo objetivou identificar o impacto dos principais tratamentos para a enxaqueca na qualidade de vida (QV) de pessoas acometidas e os instrumentos utilizados para a avaliação da QV. Trata-se de uma revisão integrativa de literatura em que foram utilizadas as bases de dados PubMed, BIREME e The Cochrane Library e selecionados artigos entre 2000 a 2009. Foram eleitos 42 artigos para análise e leitura na íntegra. Os artigos foram classificados em três categorias: tratamentos farmacológicos para a crise, tratamentos farmacológicos profiláticos e tratamentos alternativos para enxaqueca. Na maioria dos estudos os instrumentos utilizados para avaliar a QV eram validados e os mais comumente utilizados foram o Medical Outcomes Study Questionaire 36 - Item Short Form Health Survey (SF 36), o Migraine-Specific Quality of Life Questionnaire e o Migraine Disability Assessment. Conclui-se que, de uma maneira geral, os tratamentos parecem refletir positivamente na QV dos sujeitos com enxaqueca.

Descritores: Transtornos de enxaqueca; Terapêutica; Qualidade de vida.

\section{ABSTRACT}

This study aimed to identify the impact of the main treatments for migraine on the quality of life (QOL) as affected people and the instruments used to assess the QOL. It consists of an integrative review of the literature, in which the databases PubMed, BIREME and The Cochrane Library were used, considering articles from 2000 to 2009 . We selected 42 articles for analysis, which were fully read. The articles were classified into three categories: pharmacological treatments for the crisis, prophylactic drug treatments and alternative treatments for migraine. In most studies, the tools used to assess QOL were validated and the most commonly used were the Medical Outcomes Study Questionnaire 36 - Item Short Form Health Survey (SF 36), the Migraine-Specific Quality of Life Questionnaire (MSQ) and the Migraine Disability Assessment (MIDAS). We conclude that, in general, the treatments seem to be positive on the QOL of individuals with migraine.

Key words: Migraine disorders; Therapeutics; Quality of life.

\section{RESUMEN}

El objetivo de este estudio fue identificar el impacto de los principales tratamientos para la migraña en la calidad de vida de personas que sufren de esta enfermedad e identificar los instrumentos utilizados para evaluar la calidad de vida. Se trata de una revisión integradora de la literatura, en que fueran utilizadas las bases: PubMed, BIREME e The Cochrane Library, y siendo considerados los artículos publicados entre 2000 a 2009. Fueron seleccionados 42 artículos para análisis y lectura en su totalidad. Los artículos se clasificaran en tres temas: Ios tratamientos farmacológicos para la crisis, tratamientos farmacológicos profilácticos y tratamientos alternativos para la migraña. Llega-se a la conclusión de que, en general, los tratamientos parecen reflejar positivamente en la calidad de vida de los pacientes con migraña.

Palabras clave: Trastornos migranosos; Tratamiento; Calidad de vida.

\section{AUTOR CORRESPONDENTE Priscilla Hortense E-mail: prihrt@yahoo.com.br}




\section{INTRODUÇÃO}

As cefaléias são um importante problema de saúde pública no Brasil e no mundo devido ao impacto individual e social que essa condição clínica acarreta, à sua alta incidência e ao elevado potencial de cronificação, além dos custos econômicos e redução na qualidade de vida que afeta quem a sente ${ }^{(1)}$.

Em estudo realizado em Pelotas, observou-se que a prevalência de enxaqueca foi de 10,7\%, além disso, demonstrou-se que $93,5 \%$ das pessoas com enxaqueca apresentavam algum tipo de limitação nas suas atividades de vida diária na presença da dor ${ }^{(2)}$. Um estudo de revisão também apontou a alta incidência da migrânea, sendo estimada em torno de $15 \%$ na população geral, acometendo aproximadamente $20 \%$ das mulheres, $6 \%$ dos homens e $4 \%$ a $8 \%$ das crianças $^{(3)}$.

A enxaqueca, também conhecida como migrânea é uma doença neurovascular que se caracteriza por crises repetidas de dor de cabeça que podem ocorrer com uma freqüência bastante variável: enquanto alguns pacientes apresentam poucas crises durante toda a vida, outros relatam diversos episódios a cada mês. Uma crise típica de enxaqueca é reconhecida pela dor que envolve metade da cabeça, piora com qualquer atividade física e está freqüentemente associada à náusea, vômitos e desconforto com a exposição à luz e sons altos, podendo durar até $72 \mathrm{~h}$. Um conjunto de sintomas neurológicos, conhecido pelo nome de aura, costuma acompanhar o quadro de dor ${ }^{(4)}$.

Dentre as classificações das cefaléias, a migrânea é a de maior interesse devido à sua alta prevalência e seu elevado grau de comprometimento na qualidade de vida. Este estudo justifica-se devido a este alto grau de comprometimento da qualidade de vida, que ainda pode levar a queda na produtividade laboral e nas atividades da vida diária ${ }^{(3)}$, além do sofrimento físico e emocional.

\section{OBJETIVO}

Identificar o impacto dos principais tratamentos para a enxaqueca na qualidade de vida de pessoas com enxaqueca e os instrumentos utilizados para a avaliação da qualidade de vida.

\section{MÉTODO}

Trata-se de uma revisão integrativa da literatura ${ }^{(5)}$, que é um método que permite a inclusão simultânea de pesquisas experimentais e não experimentais, a fim de se obter uma ampla compreensão do fenômeno em estudo(6).

As etapas adaptadas para o desenvolvimento da revisão integrativa foram: levantamento da questão da pesquisa ou definição do problema a ser pesquisado, estabelecimento de critérios para inclusão e exclusão dos estudos, categorização dos estudos com a síntese dos principais achados e análise detalhada, interpretação dos resultados com a identificação de conclusões e implicações resultantes da revisão integrativa ${ }^{(5,6)}$.

Para que os objetivos fossem atingidos, as seguintes questões norteadoras foram delineadas: Quais são as evidências disponíveis na literatura acerca do impacto na qualidade de vida na utilização dos principais tratamentos para a enxaqueca? Que instrumentos estão sendo utilizados para a avaliação da qualidade de vida nos estudos selecionados?

Os critérios de inclusão utilizados foram: artigos que respondessem às questões norteadoras, publicados entre os anos de 2000 a 2009, redigidos em língua portuguesa, espanhola e inglesa, pesquisas com seres humanos, acima de 18 anos e artigos cujos resumos estavam apresentados na base de dados. Critérios de exclusão: artigos não encontrados na íntegra por meio da busca, revisões simples de literatura e aqueles que não respondiam às questões norteadoras propostas por este estudo.

Para a busca dos descritores padronizados, lançou-se mão do MeSH (Medical Subjects Headings) e o DeCS (Descritores em Ciências de Saúde), utilizando-se: treatment, migraine, migraine disorders e quality of life. As bases de dados bibliográficos escolhidas para a realização desta pesquisa foram: PubMed (base eletrônica ofertada pela US National Library of Medicine) pelo endereço < http://www.ncbi.nlm.nih.gov/ pubmed $>$, BIREME (Biblioteca Virtual em Saúde do Centro Latino-Americano e do Caribe de Informação em Ciências da Saúde) pelo endereço < http://regional.bvsalud.org/php/ index.php > e The Cochrane Library pelo endereço < http:// www.thecochranelibrary.com/view/0/index.html > .

A seleção dos artigos para análise foi realizada por meio da leitura do título e do resumo de todos os estudos encontrados na busca em cada uma das bases de dados. Após a seleção dos artigos, realizou-se a busca dos mesmos na íntegra por meio de serviço de comutação bibliográfica e acervo da biblioteca da Universidade Federal de São Carlos e de consulta ao Portal de Periódicos da Capes por meio do sistema de busca da Biblioteca Eletrônica da UFSCar.

Os artigos foram analisados segundo o nível e a qualidade da evidência. O nível de evidência utilizado classifica as pesquisas em seis níveis de forças de evidência segundo seu delineamento. A qualidade de qualquer nível pode variar de $A$ a $D$, sendo que cada nível reflete basicamente a credibilidade cientifica total do estudo. Uma classificação A reflete um estudo designado como muito bom e D como um estudo com grandes falhas e sérias questões sobre a credibilidade dos seus achados e é automaticamente eliminado das considerações ${ }^{(7)}$. Assim, todos os artigos foram analisados e classificados levando-se em conta: número amostral probabilístico, randomização, descrição em duplo-cego, capacidade de generalização dos resultados e vieses.

\section{RESULTADOS}

A busca nas bases de dados estabelecidas resultou em 483 artigos. Todos os títulos e resumos destes artigos foram lidos e a partir desta leitura, respeitando-se os critérios de inclusão e exclusão, foram eleitos 42 artigos para análise detalhada. Dados em detalhamento na Tabela 1.

Após a leitura e análise, os artigos elegidos foram classificados segundo sua abordagem, em três categorias: 1) MeIhora da qualidade de vida relacionada a medicamentos utilizados para o tratamento da crise da enxaqueca, 2) Melhora 
Tabela 1 - Distribuição de artigos encontrados para cada cruzamento de descritores nas diferentes bases de dados, artigos excluídos e analisados na íntegra, 2000-2009, São Carlos-SP, 2010.

\begin{tabular}{llccc}
\hline \multicolumn{1}{c}{ Base de dados } & \multicolumn{1}{c}{$\begin{array}{c}\text { Cruzamento } \\
\text { Palavras-chave }\end{array}$} & $\begin{array}{c}\text { Total de artigos } \\
\text { obtidos }\end{array}$ & $\begin{array}{c}\text { Artigos } \\
\text { Analisados } \\
\text { na íntegra }\end{array}$ \\
\hline BIREME & Migraine disorders, treatment, quality of life & 245 & 219 & 30 \\
\hline PubMed & Migraine disorders, treatment, quality of life & 175 & 24 & 25 \\
\hline $\begin{array}{l}\text { Cochrane } \\
\text { Ensaios Clínicos Controlados }\end{array}$ & Migraine disorders, quality of life & 55 & 32 \\
\hline $\begin{array}{l}\text { Cochrane } \\
\text { Revisões Sistemáticas }\end{array}$ & Migraine disorders, quality of life & 8 & 8 \\
\hline Total & & $\mathbf{4 8 3}$ & $\mathbf{4 0 7}$ \\
\hline
\end{tabular}

*34 artigos se repetiam entre as bases, sendo considerados apenas uma vez, o que resultou em 42 artigos analisados na íntegra.

da qualidade de vida relacionada a medicamentos utilizados para o tratamento profilático da enxaqueca e 3) Melhora da qualidade de vida relacionada a métodos alternativos utilizados para o tratamento da enxaqueca.

Melhora da qualidade de vida relacionada a medicamentos utilizados para o tratamento da crise da enxaqueca

Nos artigos analisados na íntegra, alguns apontaram a efetividade de drogas antimigranosas específicas: Rizatriptano $^{(8,9,10)}$, o Eletriptano(11,12), o Sumatriptano ${ }^{(13)}$ e o Almotriptano ${ }^{(14)}$, (triptanos). Outros artigos apontaram o uso do Rofecoxib (antiinflamatório) ${ }^{(15)}$ e do Proclorperazina (neuroléptico) (16). O antiinflamatório Rofecoxib foi retirado do mercado por apresentar efeitos colaterais graves e o neuroléptico Proclorperazina não é utilizado no Brasil.

Apenas um dos artigos elegidos, que compara o uso dos medicamentos Sumatriptano e Almotriptano, apontou que não houve diferenças com relação à qualidade de vida nestes dois grupos, ou seja, os dois medicamentos promovem o mesmo nível de qualidade de vida, no entanto, o estudo não possui grupo controle, o que não permite inferir se há significativa melhora da qualidade de vida em relação às pessoas que não utilizam esses tratamento ${ }^{(17)}$.

Melhora da qualidade de vida relacionada a medicamentos utilizados para o tratamento profilático da enxaqueca

Nos artigos elegidos, observou-se a efetividade do Topiramato (antiepilético e anticonvulsivante) ${ }^{(18-25)}$, do Ácido Valpróico (antiepilético e anticonvulsivante) ${ }^{(18,26)}$ dos $\beta$ bloqueadores: Nebivolol (27), Propanolol ${ }^{(18)}$, Metoprolol ${ }^{(18,27)}$ e Nadolol $^{(21)}$, dos neurolépticos ${ }^{(26)}$, do antidepressivo tricíclico Amitriptilina ${ }^{(22,26)}$, da Toxina botulínica (relaxante muscular e efeito antinocicepção) ${ }^{(25,28-30)}$, dos bloqueadores de canal de cálcio Verapamil(26) e Flunarizina $^{(18,26)}$, do Mesilato de Diidroergotamina (vasoconstritor) ${ }^{(31)}$, dos Inibidores da Recaptação Seletiva de Serotonina (IRSS) ${ }^{(18)}$, do Pizotifeno (anti-histamínico e antagonista da serotonina) ${ }^{(18)}$, dos antiinflamatórios não-esteroides $(A I N E)^{(18)}$ e do Lisinopril (inibidor de angiotensina) ${ }^{(32)}$.

Apenas um dos artigos elegidos, que compara o uso de
Toxina Botulínica e Topiramato como medicamentos profiláticos para enxaqueca, apontou que não houve diferenças com relação à qualidade de vida nestes dois grupos, ou seja, os dois medicamentos promoveram melhora significante no nível de qualidade de vida, no entanto, o estudo não possui grupo controle, o que não permite inferir se há significativa melhora da qualidade de vida em relação às pessoas que não utilizam esses tratamentos ${ }^{(25)}$.

Em dois artigos observou-se que a Toxina Botulinica não foi significantemente mais efetiva do que o placebo em melhorar a qualidade de $v^{2} a^{(33,34)}$. Outro artigo demonstrou ausência de melhorias significativas na qualidade de vida no grupo que utilizou o medicamento Candesartan (receptor bloqueador de angiotensina II) comparando ao grupo controle; os autores concluíram que o Candesartan promove efetiva profilaxia para migrânea, com um perfil de tolerabilidade comparável ao placebo. Porém, não houve diferenças significativas em relação à qualidade de vida entre os tratamentos ${ }^{(35)}$.

\section{Melhora da qualidade de vida relacionada a métodos al-} ternativos utilizados para o tratamento da enxaqueca

Os artigos elegidos demonstraram a efetividade da dieta alimentar ${ }^{(36)}$, do exercício físico ${ }^{(37-40)}$, da acupuntura ${ }^{(41-43)}$, do uso de plantas medicinais ${ }^{(44)}$, da cirurgia $^{(45,46)}$ e de uma intervenção multidisciplinar ${ }^{(47)} \mathrm{e}$ treinamento comportamental(48).

Em um artigo no qual foi avaliado o tratamento profilático com água para enxaqueca, foi identificado diminuição do número de horas de dor e da intensidade da dor no grupo água, embora esse dado não tenha sido estatisticamente significante. Não houve diferenças entre os grupos no que se refere à qualidade de vida ${ }^{(49)}$.

Na Tabela 2 é apresentada a classificação dos artigos segundo o nível de evidência e qualidade da evidência.

Observa-se que a maioria dos estudos $(59,4 \%)$ consiste em delineamento experimental, classificados com nível de evidência II. No entanto, apenas $16,6 \%$ são classificados em A na qualidade de evidência. A grande prevalência de estudos experimentais pode ser indicativa do interesse em estudos de relevância sobre tratamentos para enxaqueca, com a busca de 
Tabela 2 - Distribuição dos estudos selecionados conforme o delineamento, nível de evidência e porcentagem, 2000-2009, São Carlos-SP, 2010.

\begin{tabular}{|c|c|c|c|c|}
\hline Estudo & Delineamento & Nível de evidência & Qualidade de evidência & $\mathrm{n}(\%)$ \\
\hline $17,19,24,31,32,33,35$ & Delineamento experimental & II & A & $7(16,6)$ \\
\hline $\begin{array}{c}14,15,16,20,22,29,30,34,37,38 \\
41,42,43,45,46,47,48,49\end{array}$ & Delineamento experimental & II & B & $18(42,8)$ \\
\hline 12,21 & Delineamento quase experimental & III & A & $2(4,7)$ \\
\hline $8,9,10,11,13,18,25,26,27,40,44$ & Delineamento quase experimental & III & B & $11(26,1)$ \\
\hline 36,39 & Delineamento quase experimental & III & $\mathrm{C}$ & $2(4,7)$ \\
\hline 23,28 & Delineamento não experimental & IV & B & $2(4,7)$ \\
\hline Total & & & & 42 \\
\hline
\end{tabular}

melhores níveis de evidência, dada a magnitude deste problema do ponto de vista epidemiológico.

Para a avaliação da qualidade de vida relacionada aos tratamentos para enxaqueca, a maioria dos autores utilizou instrumentos validados. Alguns autores utilizaram mais de um instrumento e outros utilizaram apenas escalas visuais analógicas direcionadas à qualidade de vida. Observou-se a utilização dos seguintes instrumentos para avaliação do impacto na qualidade de vida relacionada à saúde: SF 36 (Medical Outcomes Study Questionaire 36 - Item Short Form Health Survey), SF 12, MSQ (Migraine-Specific Quality of Life Questionnaire), MQOLQ (Migraine Quality of Life Questionnaire), MIDAS (Migraine Disability Assessment), HIT 6 (Headache Impact Test), PDI (Pain Disability Index) e 24-HrMQoLQ (24-Hour Migraine-Specific Quality of Life Questionnaire).

Em treze dos quarenta e dois artigos analisados, foi utilizado o SF $36^{(9,18,21,26-27,32-33,35,41-43,45,48)}$, instrumento validado ${ }^{(50)}$ que consiste em oito subcategorias do conceito de saúde: funcionamento físico, funcionamento social, dor corporal, vitalidade, papel emocional e físico na vida, saúde geral e saúde mental. Cada subcategoria é avaliada por meio de várias perguntas. Os respondentes têm de escolher entre diferentes respostas, que variam de muito boa a muito ruim e muita dificuldade a sem dificuldade. A pontuação é de 0 a 100 e um valor numérico final irá indicar o índice de qualidade de vida. Em um artigo ${ }^{(24)}$ foi utilizada a escala validada ${ }^{(51)}$ SF 12 que reproduz uma síntese do componente físico e mental do SF-36.

Em onze artigos foi utilizado o MSQ ${ }^{(13,19-22,31,33-34,36,40,48)}$, que também é um instrumento validado(52). Este instrumento é dividido em três dimensões: restrição de função, prevenção de função e função emocional. Cada domínio é constituído de várias perguntas e os respondentes devem escolher entre diferentes respostas, as quais variam de nenhum dos tempos até o tempo todo. O valor numérico final varia de 0 a 100, sendo que, quanto mais próximo de 100 , maior o nível de qualidade de vida.

Em oito artigos foi utilizado o MQOLQ ${ }^{(8,10-12,14-17)}$, este instrumento é validado ${ }^{(53)}$ e constituído de 15 questões sobre qualidade de vida no período das últimas 24 horas no que se refere ao tratamento da crise de enxaqueca. As questões abordam a capacidade de fazer trabalho diário normal, a de gozar a vida, o nível de energia, a capacidade de ter uma boa noite de sono e humor, sintomas da enxaqueca e preocupação. Com categorias de respostas que variam de "o tempo todo" para "nenhum dos tempos". A pontuação varia de 0 a 105 pontos. Um valor numérico final irá indicar o índice de qualidade de vida.

Emtrezeartigosfoi utilizadooMIDAS ${ }^{(14,18-20,24,25,27,29,30,39,45,46,48)}$. Trata-se de um instrumento validado ${ }^{(54)}$, no qual existem cinco questões relacionadas com o tempo perdido por causa da migrânea, relacionado à escola ou trabalho, ao trabalho doméstico e às atividades familiares e sociais, além de duas questões adicionais sobre frequência e intensidade de dor, as quais não estão incluídas na pontuação. A pontuação varia de 0 a 21 e quanto maior a pontuação maior a incapacidade/deficiência.

Em três artigos foi utilizado o HIT-6 ${ }^{(23,25,29)}$, instrumento validado $^{(55)}$ que apresenta uma série de seis questões, abrangendo as áreas de dor, capacidade de realizar atividades usuais, funcionamento social, energia/fadiga, cognição e estresse emocional. É considerada uma medida válida, confiável e precisa para medir o impacto da dor de cabeça. A pontuação varia entre 36 a 78 pontos e quanto maior a pontuação obtida, maior o impacto da dor de cabeça na vida da pessoa.

Em dois artigos foi utilizado o PDI ${ }^{(37,42)}$. O PDI é um instrumento validado ${ }^{(56)}$, simples e rápido para avaliar o impacto que a dor tem na habilidade da pessoa em realizar atividades essenciais na vida. Ele pode ser utilizado para avaliar os pacientes ao longo do tempo para julgar a efetividade das intervenções. A pontuação varia de 0 a 70 pontos e quanto maior pontuação obtida, maior a incapacidade para realizar atividades devido à dor.

O 24-HrMQoLQ foi utilizado em um artigo ${ }^{(10)}$. Trata-se de um instrumento validado ${ }^{(57,58)}$ que avalia o impacto na qualidade de vida 24 horas após terapia para ataque agudo de enxaqueca. Esse questionário consiste de 15 questões distribuídas em domínios: funcionamento no trabalho, funcionamento social, energia/vitalidade, sintomas e sentimentos/preocupações. As pontuações dos domínios variam de 3 (pior funcionamento possível/sintomas de enxaqueca o tempo todo) a 21 (melhor funcionamento possível/sem sintomas).

Em dois artigos foram utilizadas Escalas Analógicas Visuais $(44,47)$. Estas consistem em uma linha com escores que variam 
de 0 ("extremamente ruim) a 10 ("muito bom"), sendo que o paciente deveria avaliar sua qualidade de vida se posicionando em algum ponto desta escala. Nos demais artigos foram utilizados instrumentos não validados e escala Likert, além de um artigo em que foi utilizado um instrumento alemão de avaliação de qualidade de vida, e outro em que foi usada a Headache Pain Specific Quality of Life measure ${ }^{(28,30,33,37,38)}$.

\section{DISCUSSÃO}

Um consenso realizado em 2000 apresenta as recomendações para o tratamento da crise migranosa. Os grupos farmacológicos recomendados são: Ácido acetilsalicílico, Paracetamol, Dipirona, AINEs, Ergotamina, DHE (di-hidroergotamina), Clonixilato de lisina, Indometacina, Clorpromazina, Dexametasona, Haloperidol, Noratriptano, Sumatriptano, Zomitriptano e Rizatriptano ${ }^{(59)}$. Os resultados obtidos nesta revisão de literatura apontam que, para a crise migranosa, o uso dos triptanos melhoram a qualidade de vida, assim como um AINE ${ }^{(15)}$ que foi proibido no Brasil e um neuroléptico ${ }^{(16)}$ que não é usado neste país.

Em um dos estudos os autores apontam que o nível de qualidade de vida foi significantemente maior no grupo que utilizou Rizatriptano para as crises agudas do que aqueles tratados com terapias usuais ${ }^{(8)}$. Em outro estudo também foi encontrado melhora significativa na qualidade de vida nas 24 horas após a crise aguda de enxaqueca no grupo tratado com Rizatriptano em comparação com outros tratamentos convencionais $^{(10)}$. O tratamento com Rizatriptano melhorou significantemente o absenteismo e a produtividade no trabalho, a redução nos dias perdidos de trabalho foi de $60 \%$. O uso de serviço médico, clinica de dor e serviços de emergência também reduziu significantemente e ainda ocorreu significante melhora em todos os domínios da qualidade de vida ${ }^{(9)}$.

O tratamento com Eletriptano foi associado a altos níveis de satisfação em 2/3 dos pacientes no que se refere ao grau de alívio da intensidade da dor, no tempo de alívio de dor, na duração do alívio, no tempo de retorno para atividades usuais e no alívio de sintomas associados. A elevada satisfação relatada também foi refletida no aumento na qualidade de vida e na melhora da funcionalidade ${ }^{(11)}$.

Já em outro estudo, realizado com a utilização do medicamento Sumatriptano, os sujeitos perceberam melhora em sua habilidade de cuidar da família e amigos, na concentração no trabalho e nas atividades diárias, além de perceberem redução na necessidade de cancelar ou parar o trabalho ou atividades diárias por causa das crises de enxaqueca, requisitaram menos ajuda e sentiram-se com maior capacidade de aproveitar atividades de lazer, ir às atividades sociais, possibilitando menor frustração com relação às crises de enxaqueca ${ }^{(13)}$.

O tratamento logo no início da crise com Almotriptano foi associado com maiores pontuações em todos os domínios da qualidade de vida, possibilitando a redução do impacto na vida associado à enxaqueca ${ }^{(14)}$.

Assim, dos nove artigos encontrados na literatura sobre a melhora na qualidade de vida após tratamento das crises de enxaqueca, sete $(77,8 \%)$ são artigos que avaliaram e demonstraram eficácia no uso dos triptanos.

Nesta revisão, observou-se uma grande quantidade de estudos para avaliar a efetividade no uso de drogas profiláticas para enxaqueca. Os medicamentos encontrados foram Topiramato, Ácido Valpróico, Nebivolol, Propranolol, Metoprolol, Nadolol, neurolépticos, Amitriptilina, Toxina Botulínica, Verapamil e Flunarizina. Um consenso de 2002 apresenta recomendações para o tratamento profilático da enxaqueca, sendo recomendados: beta-bloqueadores, antidepressivos, bloqueadores dos canais de cálcio, antagonistas da serotonina, antiepilépticos e miscelânea (drogas dopaminérgicas, antiagregantes plaquetários, anticoagulantes, co-fatores do metabolismo da serotonina -riboflavina- e toxina botulínica) ${ }^{(60)}$.

Foi afirmado em um estudo que o Topiramato contribui para a melhora das atividades da vida diária, qualidade de vida e redução da angústia em pacientes com enxaqueca crônica $^{(22)}$. Autores também afirmam que o Nebivolol, um seletivo beta bloqueador com efeito profilático na enxaqueca que é similar ao Metoprolol em termos de prevenção de ataque ${ }^{(27)}$. Em outro estudo, a utilização de medicação preventiva com Nadolol ou com Topiramato também resultou em impacto positivo na qualidade de vida dos pacientes com enxaqueca ${ }^{(21)}$.

A Toxina botulínica demonstrou consistente efetividade na redução da enxaqueca associada à incapacidade. Além de ter reduzido o impacto nas atividades da vida diária, melhorou a satisfação com o tratamento e a qualidade de vida ${ }^{(29)}$. Em outro estudo, foi demonstrado que a Toxina botulínica trouxe uma significante redução de episódios de enxaqueca e de dias com enxaqueca, além de melhora da qualidade de vida, se comparada ao placebo ${ }^{(30)}$. O uso de injeções de toxina botulínica no manejo da enxaqueca em pacientes que falharam com outras terapias também foi associado a uma melhora na qualidade de vida na maioria desses pacientes ${ }^{(28)}$.

De modo geral, o uso de tratamentos profiláticos com medicamentos demonstrou redução na pontuação total da qualidade de vida, indicando significante melhoria na habilidade de funcionamento em diferentes atividades da vida diária, com um decréscimo no número de dias perdidos ou comprometidos nas atividades gerais e de trabalho. A Melhoria na qualidade de vida avaliada pelo SF-36 também foi demonstrada, com significante aumento nos escores das escalas que exploravam o grau de interferência da saúde física nas atividades sociais, de trabalho e pessoais, sentido geral de bem-estar e controle comportamental e emocional ${ }^{(18)}$.

Uma associação entre melhoria da qualidade de vida com a redução na freqüência de enxaqueca foi sugerida, o que pode indicar que a melhora na qualidade de vida na presença de enxaqueca é mediada, pelo menos em parte, pela redução na freqüência dos ataques ${ }^{(21)}$.

O presente estudo possibilitou identificar que, dos artigos analisados sobre tratamento profilático para enxaqueca, a maioria avaliou o uso do Topiramato, dos beta-bloqueadores e da Toxina Botulínica. Essas drogas foram estudadas em $73,3 \%$ dos estudos analisados, que efetivamente demonstraram o impacto positivo das mesmas na prevenção da enxaqueca e consequente melhora na qualidade de vida. Vale ressaltar que, mesmo em menor proporção, os tratamentos com 
Ácido Valpróico, Neurolépticos, Amitriptilina, Bloqueadores de Ca, Tizanidina, Dicloroergotamina, IRSS, AINE e Lisinopril também apresentaram impacto positivo na qualidade de vida.

Com relação aos tratamentos alternativos tidos como eficazes para a melhora da qualidade de vida no paciente com enxaqueca, foram encontrados nesta revisão: a dieta alimentar, a intervenção multidisciplinar, a cirurgia, os exercícios físicos e o relaxamento, uso de plantas medicinais e o treinamento comportamental. Um consenso da Sociedade Brasileira de Cefaleia, do ano de 2002, também recomenda o uso de tratamentos não farmacológicos para a profilaxia de enxaqueca como biofeedback, técnicas de relaxamento, terapia cognitiva comportamental, dieta, acupuntura, psicoterapia e fisioterapia ${ }^{(60)}$.

Um estudo foi realizado com base na filosofia naturopática médica, que trata a doença como uma deterioração da função normal do organismo. Esta filosofia considera que o organismo não recebe o que precisa por meio da alimentação, devido à assimilação defeituosa ou à incapacidade do corpo em remover resíduos e toxinas. Este estudo teve o objetivo de eliminar os obstáculos da cura concentrando-se em melhorar a assimilação e eliminação e permitindo que habilidades inerentes de reparação e recuperação do corpo funcionem adequadamente. Os autores concluem que com o uso de terapia nutricional é possível obter uma significante melhora da qualidade de vida em pessoas com enxaqueca ${ }^{(36)}$.

O exercício físico também parece reduzir a frequência e a severidade da enxaqueca. Os sujeitos de um dos estudos declararam que o exercício trouxe tranqüilidade, relaxamento e bom humor e ainda diminuiu os ataques de dor e a incapacidade, possibilitando assim melhora na qualidade de vida ${ }^{(37)}$.

A intervenção de um grupo multidisciplinar (neurologista para confirmação do diagnóstico e critérios de inclusão, fisioterapeuta, terapia com exercício supervisionado, grupo de palestras com psicólogo, grupo de palestras com nutricionista e sessões de massagem terapêutica) foi efetiva em reduzir a freqüência, a intensidade e a duração da dor, assim como a incapacidade e a depressão. Foi observado nos sujeitos aumento da auto-percepção da condição funcional, da qualidade de vida e da condição de saúde ${ }^{(47)}$.
O treinamento comportamental, técnica realizada para a profilaxia da enxaqueca e que utiliza-se dos princípios da Psicologia comportamental, foi realizado em um estudo com treinadores leigos que apresentavam enxaqueca. Os resultados obtidos mostraram melhora da qualidade de vida no grupo que recebeu o treinamento comportamental em relação ao grupo controle ${ }^{(48)}$.

Ressalta-se a importância de métodos alternativos profiláticos para a melhora da qualidade de vida das pessoas com enxaqueca. No entanto, entende-se que há a necessidade de mais estudos que demonstrem esta importância.

Nos artigos analisados percebe-se uma preocupação na utilização de instrumentos validados para a avaliação do impacto dos tratamentos na qualidade de vida das pessoas com enxaqueca, já que o aperfeiçoamento e a criação de instrumentos de observação e medida foram e são responsáveis por muitas descobertas científicas. De fato, para que ocorra avanço do conhecimento é necessário que os instrumentos sejam considerados fidedignos e válidos. Fidedignidade no seu poder de replicação e consistência dos resultados e validade para que os resultados representem a realidade da variável que foi mensurada ${ }^{(61)}$.

\section{CONCLUSÃO}

A enxaqueca ocasiona um alto grau de incapacidade, gerando queda na qualidade de vida das pessoas. Os estudos encontrados nesta revisão apontam para uma tendência na investigação da qualidade de vida dos sujeitos com enxaqueca a partir de tratamentos farmacológicos para a crise, farmacológicos profiláticos e tratamentos alternativos. Observa-se também a utilização de diferentes instrumentos validados para a identificação da qualidade de vida dos sujeitos e ainda, uma maior prevalência de método experimental e quase experimental para atingir os objetivos propostos, o que sinaliza para uma tendência na busca de melhores evidências científicas no tratamento da enxaqueca, guardadas as devidas exigências metodológicas que estas modalidades de estudos requerem para produzir resultados fidedignos. De uma maneira geral, os tratamentos parecem refletir positivamente na qualidade de vida dos sujeitos com enxaqueca.

\section{REFERÊNCIAS}

1. Galdino GS. Cefaléias primárias: abordagem diagnóstica por médicos não-neurologistas. Arq. Neuropsiquiatr 2007;65(3-a):681-4.

2. Pahim LS, Menezes AMB, Lima R. Prevalência e fatores associados à enxaqueca na população adulta de Pelotas, RS. Rev Saúde Pública 2006;40(4):692-8.

3. Morais MSBBF, Benseñor IM. Cefaléias primárias. Rev Bras Med 2009;66(6):138-47.

4. Sociedade Brasileira de Cefaléia [homepage na internet] Tipos de dor de cabeça. [acesso $30 \mathrm{dez} 2010]$. Disponível em: < http://www.sbce.med.br/tudo-o-que-voce-queria-saber/ news/dor-de-cabeca/tipos-de-dor-de-cabeca >

5. Broome ME. Integrative literature reviews for the development of concepts. In: RODGERS, B.L.; KNAFL, K.A., editors. Concept development in nursing: foundations, techniques and applications. Philadelphia: W.B Saunders Company; 2000. p.231-50.

6. Whittemore $R$, Knafl K. The integrative review: updated methodology. J Adv Nurs 2005;52(5):546-53.

7. Stetler CB, Morsi D, Rucki S, Broughton S, Corrigan $B$, Fitzgerald J et al. Utilization-focused integrative reviews in a nursing service. Appl Nurs Res 1998;11(4):195-206. 
8. Baos V, Serrano A, Torrecilla M, Bertral C, Caloto MT, Nocea $\mathrm{G}$ et al. Patient-reported benefits of rizatriptan compared with usual non-triptan therapy for migraine in a primary care setting. Int J Clin Pract 2003;57(9):761-8.

9. Láinez MJ, López A, Pascual AM. Effects on productivity and quality of life of rizatriptan for acute migraine: a workplace study. Headache 2005;45(7):883-90.

10. Gerth WC, Ruggles KH, Stark SR, Davies GM, Santanello NC. Improvement in Health-Related Quality of Life with Rizatriptan 10mg Compared with Standard Migraine Therapy. Clin Drug Investg 2001;21 (12):853-60.

11. Nett RB, Tiseo PJ, Almas M, Sikes CR. Patient satisfaction with eletriptan in the acute treatment of migraine primary care. Int J Clin Pract 2007;61(10):1677-85.

12. Mérelle SYM, Couturier EGM, van Bokhorst J, Busschbach JJV, Passchier J. Large-scale screening and subsequent effects of migraine treatment on the work floor in the Netherlands. Cephalalgia 2009;29(6): 606-15.

13. Smith $T$, Blumenthal $H$, Diamond $M$, Mauskop A, Ames M, McDonald S, et al. Sumatriptan/Naproxen sodium for migraine: efficacy, health related quality of life, and satisfaction outcomes. Headache 2007; 47(5):683-92.

14. Freitag F, Smith T, Mathew N, Rupnow M, Greenberg $S$, Mao L, et al. Effect of early intervention with almotriptan vs placebo on migraine-associated functional disability: results from the AEGIS Trial. Headache 2008;48(3):341-54.

15. Silberstein S, Tepper S, Brandes J, Diamond M, Goldstein J, Winner $\mathrm{P}$, et al. Randomized, placebo-controlled trial of rofecoxib in the acute treatment of migraine. Neurol 2004;62(9):1552-7.

16. Sharma S, Prasad A, Nehru R, Anand KS, Rishi RK, Chaturvedi S, et al. Efficacy and tolerability of prochlorperazine buccal tablets in treatment of acute migraine. Headache 2002;42(9):896-902.

17. Colman SS, Brod MI, Krishnamurthy A, Rowland CR, Jirgens KJ, Gomez-Mancilla B. Treatment satisfaction, functional status, and health-related quality of life of migraine patients treated with almotriptan or sumatriptan. Clin Ther 2001;23(1):127-45.

18. D'Amico D, Solari A, Usai S, Santoro P, Bernardoni P, Frediani $F$, et al. Improvement in quality of life and activity limitations in migraine patients after prophylaxis. A prospective longitudinal multicentre study. Cephalalgia 2006;26(6):691-6.

19. Diener HC, Bussone G, Van Oene JC, Lahaye M, Schwalen S, Goadsby PJ, et al. Topiramate reduces headache days in chronic migraine: a randomized, double-blind, placebocontrolled study. Cephalalgia 2007;27(7):814-23.

20. Dodick DW, Silberstein S, Saper J, Freitag FG, Cady RK, Rapoport AM, et al. The impact of topiramate on health-related quality of life indicators in chronic migraine. Headache 2007;47(10):1398-408.

21. Garcia-Monco JC; Foncea N; Bilbao A; Ruiz de Velasco I; Gomez-Beldarrain M. Impact of preventive therapy with nadolol and topiramate on the quality of life of migraine patients. Cephalalgia 2007; 27(8):920-8.

22. Dodick DW, Freitag F, Banks J, Saper J, Xiang J, Rupnow
$M$, et al. Topiramate versus amitriptyline in migraine prevention: a 26-week, multicenter, randomized, doubleblind, double-dummy, parallel-group noninferiority trial in adult migraineurs. Clin Ther 2009;31(3):542-59.

23. Nelles G, Schmitt L, Humbert T, Becker V, Sandow P, Bornhoevd $K$, et al. Prevention of episodic migraines with topiramate: results from a non-interventional study in a general practice setting. J Headache Pain 2009;11(1):33-44.

24. Diener HC, Agosti R, Allais G, Bergmans P, Bussone $G$, Davies $B$ et al. Cessation versus continuation of 6-month migraine preventive therapy with topiramate (PROMPT): randomised, double-blind, placebo-controlled trial. Lancet Neurol 2007;6(12):1054-62

25. Mathew NT, Jaffri SF. A double-blind comparison of onabotulinumtoxina (BOTOX) and topiramate (TOPAMAX) for the prophylactic treatment of chronic migraine: a pilot study. Headache 2009;49(10):1466-78.

26. Bordini CA, Mariano da Silva H, Garbelini RP, Teixeira SO, Speciali JG. Effect of preventive treatment on healthrelated quality of life in episodic migraine. J Headache Pain 2005;6(5):387-91.

27. Schellenberg R, Lichtenthal A, Wöhling H, Graf C, Brixius K. Nebivolol and metoprolol for treating migraine: an advance on beta-blocker treatment? Headache 2008;48(1):118-25.

28. Mitchell MP, Schaecher K, Cannon HE, Speckman M. Humanistic, utilization, and cost outcomes associated with the use of botulinum toxin for treatment of refractory migraine headaches in a managed care organization. J Manag Care Pharm 2008;14(5):442-50.

29. Cady R, Schreiber C. Botulinum toxin type A as migraine preventive treatment in patients previously failing oral prophylactic treatment due to compliance issues. Headache 2008;48(6):900-13.

30. Freitag FG, Diamond S, Diamond M, Urban G. Botulinum Toxin Type $A$ in the treatment of chronic migraine without medication overuse. Headache 2008;48(2):201-9.

31. Pradalier A, Lantéri-Minet M, Géraud G, Allain $H$, Lucas C, Delgado A. The PROMISE study: PROphylaxis of MIgraine with SEglor (dihydroergotamine mesilate) in French primary care. CNS Drugs 2004;18(15):1149-63.

32. Schrader H, Stovner LJ, Helde G, Sand T, Bovim G. Prophylactic treatment of migraine with angiotensin converting enzyme inhibitor (lisinopril): randomised, placebo controlled, crossover study. BMJ 2001;322(7277):19-22.

33. Elkind $\mathrm{AH}, \mathrm{O}^{\prime}$ Carroll $\mathrm{P}$, Blumenfeld A, DeGryse R, Dimitrova R; BoNTA-024-026-036 Study Group. A series of three sequential, randomized, controlled studies of repeated treatments with botulinum toxin type A for migraine prophylaxis. J Pain 2006;7(10):688-96.

34. Vo AH, Satori R, Jabbari B, Green J, Killgore WD, Labutta $\mathrm{R}$, et al. Botulinum toxin type-a in the prevention of migraine: a double-blind controlled trial. Aviat Space Environ Med 2007;78 Suppl 5:B113-8.

35. Tronvik E, Stovner LJ, Helde G, Sand T, Bovim G. Prophylactic treatment of migraine with an angiotensin II receptor blocker: a randomized controlled trial. JAMA 2003;289(1):65-9. 
36. Sensenig J, Johnson M, Staverosky T. Treatment of migraine with targeted nutrition focused on improved assimilation and elimination. Altern Med Rev 2001;6(5):488-94.

37. Narin SO, Pinar L, Erbas D, Oztürk V, Idiman F. The effects of exercise and exercise-related changes in blood nitric oxide level on migraine headache. Clin Rehabil 2003;17(6):624-30.

38. Dittrich SM, Günther V, Franz G, Burtscher M, Holzner $B$, Kopp M. Aerobic exercise with relaxation: influence on pain and psychological well-being in female migraine patients. Clin J Sport Med 2008; 18(4):363-5.

39. Elinoff V, Lynn SJ, Ochiai H, Hallquist M. The efficacy of Kiko exercises on the prevention of migraine headaches: a pilot study . Am J Chin Med 2009;37(3):459-70.

40. Varkey E, Cider A, Carlsson J, Linde M. A study to evaluate the feasibility of an aerobic exercise program in patients with migraine. Headache 2009;49(4):563-70.

41. Vickers AJ, Rees RW, Zollman CE, McCarney R, Smith $\mathrm{CM}$, Ellis N, et al. Acupuncture of chronic headache disorders in primary care: randomised controlled trial and economic analysis. Health Technol Assess 2004;8(48):1-35.

42. Melchart D, Weidenhammer W, Streng A, Hoppe A, Pfaffenrath V, Linde K. Acupuncture for chronic headaches--an epidemiological study. Headache 2006;46(4):632-41.

43. Witt CM, Reinhold T, Jena S, Brinkhaus B, Willich SN. Cost-effectiveness of acupuncture treatment in patients with headache. Cephalalgia 2008;28(4):334-45.

44. Shrivastava R, Pechadre JC, John GW. Tanacetum parthenium and Salix alba (Mig-RL) combination in migraine prophylaxis: a prospective, open-label study. Clin Drug Investig 2006;26(5):287-96.

45. Guyuron B, Reed D, Kriegler JS, Davis J, Pashmini $\mathrm{N}$, Amini S. A placebo-controlled surgical trial of the treatment of migraine headaches. Plast Reconstr Surg 2009;124(2):461-8.

46. Kimmelstiel C, Gange C, Thaler D. Is patent foramen ovale closure effective in reducing migraine symptoms? A controlled study. Catheter Cardiovasc Interv 2007; 1;69(5):740-6.

47. Lemstra M, Stewart B, Olszynski WP. Effectiveness of multidisciplinary intervention in the treatment of migraine: a randomized clinical trial. Headache 2002;42(9):845-54.

48. Mérelle SY, Sorbi MJ, van Doornen LJ, Passchier J. Lay trainers with migraine for a home-based behavioral training: a 6-month follow-up study. Headache 2008;48(9):1311-25.
49. Spigt MG, Kuijper EC, Schayck CP, Troost J, Knipschild PG, Linssen VM et al. Increasing the daily water intake for the prophylactic treatment of headache: a pilot trial. Eur J Neurol 2005;12(9):715-8.

50. Ware JE Jr, Sherbourne CD. The MOS 36-item short-form health survey (SF-36). I. Conceptual framework and item selection. Med Care 1992;30:473-83.

51. Ware J Jr, Kosinski M, Keller SD. A 12-item short-form health survey: construction of scales and preliminary tests of reliability and validity. Med Care 1996;34:220-33.

52. Patrick DL, Hurst BC, Hughes J. Further development and testing of the migraine-specific quality of life (MSQOL) measure. Headache 2000;40(7):550-60.

53. Santanello NC. Validation of a new quality of life questionnaire for acute migraine headache. Headache 1995;35:330-7.

54. Stewart WF, Lipton RB, Dowson AJ, Sawyer J. Development and testing of the Migraine Disability Assessment (MIDAS) Questionnaire to assess headache-related disability. Neurol 2001;56 Suppl 1:S20-8.

55. KosinskiM,BaylissMS,Bjorner JB, et al. A six-item shortform survey for measuring headache impact: The HIT-6. Qual Life Res 2003;12:963-74.

56. Chibnall JT, Tait RC. The pain disability index: factor structure and normative data. Arch Phys Med Rehabil 1994;75:1082-6.

57. Hartmaier SL, Santanello NC, Epstein RS, Silberstein SD. Development of a brief 24-hour migraine-specific quality of life questionnaire. Headache 1995;35(6):320-9.

58. Santanello NC, Hartmaier SL, Epstein RS, Silberstein SD. Validation of a new quality of life questionnaire for acute migraine headache. Headache 1995;35(6):330-7.

59. Sociedade Brasileira de Cefaléia. Recomendações para o tratamento da crise migranosa. Arq. Neuropsiquiatr [periódico na internet]. 2000 Jun [acesso em 20 Nov 2010] Disponível em: <http://www.scielo.br/scielo. php?pid = S0004-82X2000000200029\&script $=$ sci_arttext>

60. Sociedade Brasileira de Cefaléia. Recomendações para o tratamento profilático da migrânea. Arq Neuropsiquiatr [periódico na internet]. 2002 Mar [acesso em $20 \mathrm{Nov}$ 2010];60(1) Disponível em: < http://www.scielo.br/scielo.php?script $=$ sci_arttext\&pid $=$ S0004-282X200200010 0030\&lng $=$ en\&nrm $=$ iso $>$

61. Faleiros Sousa, F.; Da Silva, J. A. A métrica da dor (dormetria): problemas teóricos e metodológicos. Rev Dor 2005;6(1):469-88. 